\title{
Construction of a scoring system for predicting the risk of severe gastrointestinal involvement in Henoch-Schönlein Purpura
}

Tsunehisa Nagamori ${ }^{1{ }^{*}+}$, Hideharu Oka ${ }^{2 \dagger}$, Shin Koyano ${ }^{1}$, Hironori Takahashi ${ }^{1}$, Junichi Oki ${ }^{2}$, Yuko Sato ${ }^{3}$, Koichi Murono ${ }^{3}$, Kenichi Iseki ${ }^{4}$, Ryou Takeguchi ${ }^{5}$, Takahiro Takeda ${ }^{5}$, Masayuki Sato ${ }^{6}$, Rika Sugai ${ }^{6}$, Hiroyuki Kitamura ${ }^{7}$, Hiroki Kajino ${ }^{7}$, Yurika Miura ${ }^{8}$, Toru Ishioka ${ }^{8}$ and Hiroshi Azuma'

\begin{abstract}
Objective: To evaluate the parameters associated with significant gastrointestinal (GI) involvement in Henoch-Schönlein Purpura (HSP), and construct a scoring system for the identification of patients at high risk of gross blood in stools.

Study design: Data for HSP patients hospitalized at each of seven institutes were retrospectively analyzed. Patients were divided into four groups according to the consequent severity of Gl involvement. Identification of laboratory parameters at the time of admission were then used to differentiate the groups, and a scoring system to predict gross intestinal bleeding was constructed. Prognostic efficiency, correlation with the subsequent duration of abdominal pain, and association with manifestations excluding abdominal pain were also analyzed.

Results: An analysis of variance (ANOVA) test showed significant intergroup differences in white blood cell (WBC) count, neutrophil count, serum albumin, potassium, plasma D-dimer and coagulation factor XIII activity. A scoring system consisting of these parameters showed a good prognostic value for gross intestinal bleeding in a receiver operating characteristic (ROC) analysis, and a cut-off value of 4 points showed a sensitivity of $90.0 \%$ and specificity of $80.6 \%$. The score was also correlated with the duration of abdominal pain after admission. A significantly higher score (s) was observed in patients presenting with nephritis, although the predictive value was poor.
\end{abstract}

Conclusion: A scoring system consisting of generally available parameters was of use in predicting severe Gl involvement in HSP patients. Although further study is needed, initial therapy in accordance with disease activity may be taken into consideration using this scoring system.

\section{Introduction}

Henoch-Schönlein Purpura (HSP) is a systemic, IgAmediated, small vessel vasculitis which is common in children (Saulsbury 2001). Clinical manifestations including non-thrombocytopenic purpura, gastrointestinal (GI) involvement and arthralgia are common in the early phase of HSP. Further, HSP nephritis (HSPN), the onset of which may be delayed for weeks or months after the appearance of other symptoms, is the most serious

\footnotetext{
* Correspondence: ntsune5p@asahikawa-med.ac.jp

${ }^{\dagger}$ Equal contributors

'Department of Pediatrics, Asahikawa Medical University, Asahikawa,

Hokkaido, Japan

Full list of author information is available at the end of the article
}

long-term complication of HSP (Narchi 2005). Although HSP generally follows a benign, self-limiting course that is resolved within a few weeks, some cases present with severe GI involvement including massive intestinal bleeding that can lead to acute complications such as intestinal perforation or obstruction (Choong \& Beasley 1998; Saulsbury 2007). Severe GI involvement presenting as gross blood in stools in the early phase of HSP is important, not only because of the distress it causes patients, but also because it is a significant risk factor for the subsequent onset of HSPN (Kaku et al. 1998; Sano et al. 2002; Bogdanovic 2009). However, the assessment of GI involvement can be often difficult due 
to the nature of the abdominal pain. It is colicky and often fluctuates, and the common presentation of blood in stools can be delayed for several days after the onset of other symptoms (Zhang \& Huang 2008).

Previous reports have already demonstrated that HSP patients present with a high serum IgA concentration (Saulsbury 1999; Calvino et al. 2001; Trapani et al. 2005), low C3 or C4 (Calvino et al. 2001; Trapani et al. 2005), and leukocytosis (Trapani et al. 2005). Furthermore, a decrease in fXIII activity (Kamitsuji et al. 1987), and increases in prothrombin fragment $1+2$ and D-dimer levels (Yilmaz et al. 2005) were shown to be associated with the severity of GI involvement. Unfortunately, however, the sole predictive value of these parameters for intestinal bleeding has not yet been demonstrated.

In the present study, we conducted a multi-centered retrospective analysis of 140 patients with HSP to evaluate the parameters associated with significant GI involvement, and construct a scoring system for the identification of patients at high risk for such involvement.

\section{Patients and methods}

The study was performed in accordance with the ethical standards laid down in the Declaration of Helsinki principles.

\section{Study population}

We retrospectively reviewed the medical records of HSP patients hospitalized between January 2003 and December 2012 at seven institutions in Hokkaido, Japan. A diagnosis of HSP was made on the basis of the presence of the major manifestations of the illness, consisting of purpura and abdominal pain or arthralgia without thrombocytopenia (Saulsbury 1999). HSPN was defined by the presence of gross or microscopic hematuria ( $>5$ cells per high power field from a centrifuged specimen) either with or without proteinuria (Saulsbury 1993). Patients were excluded on the basis of severe complications affecting abdominal pain or a lack of laboratory data. Patients representing with abdominal pain later than two days after hospitalization were also excluded, in consideration of

Table 1 Baseline characteristics and gastrointestinal involvement (GI) groups

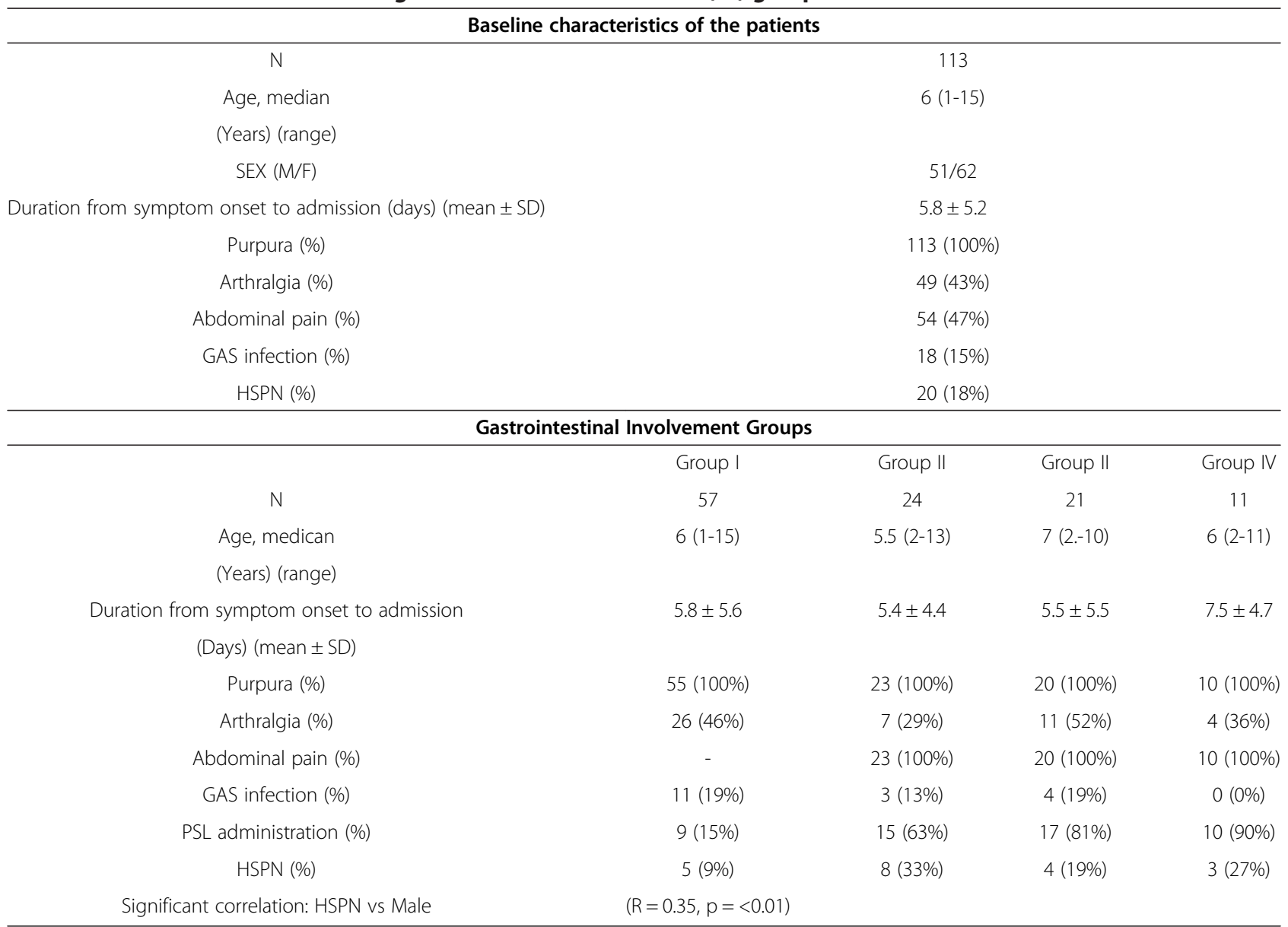

Gl groups were categorized as follows; Group I: no abdominal pain, Group II: abdominal pain without fecal hemoglobin, Group III: abdominal pain and positive for fecal hemoglobin, but negative for gross blood in stools, and Group IV: presence of gross blood in stools. 
their laboratory data not reflecting actual disease activity. Fecal hemoglobin was repetitively examined as long as abdominal pain continued. The duration of abdominal pain after admission was defined as the number of hospitalization day(s) the patient suffered from abdominal pain without remission for a 24-hour interval.

\section{Therapeutic strategy for early phase HSP}

The essential therapeutic strategy for early phase HSP on admission was basically concordant among the participating institutes in that patients were recommended bed rest, and oral or intravenous prednisolone (PSL) $1.5 \sim 2 \mathrm{mg} / \mathrm{kg} /$ day was administrated when the patient complained of unacceptable abdominal or joint pain.

\section{Four patient groups categorized according to gastrointestinal involvement}

Patients were divided into four groups based on the severity of gastrointestinal (GI) involvement as follows; Group I: no abdominal pain, Group II: presence of abdominal pain, without intestinal bleeding (negative

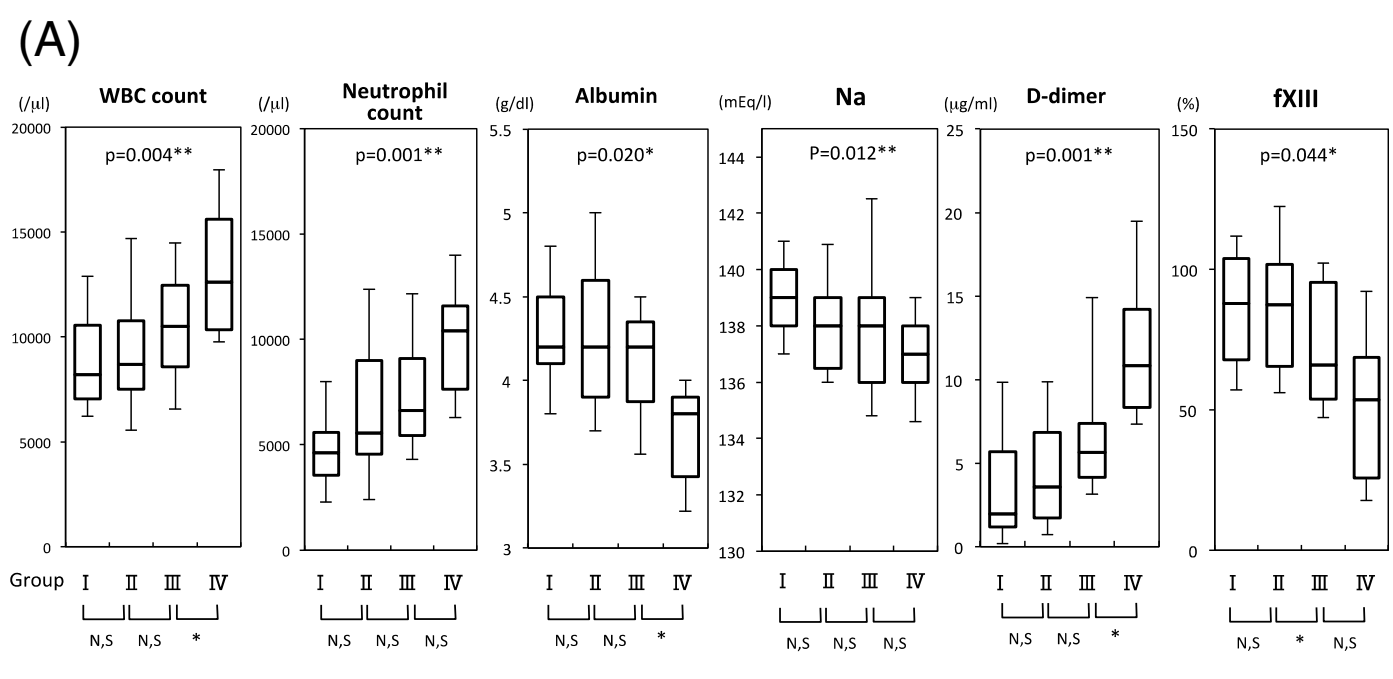

(B)

\begin{tabular}{|lll|}
\hline \multirow{2}{*}{ WBC } & $>10000 / \mu \mathrm{l}$ & $\mathbf{1}$ \\
& $>15000 / \mu \mathrm{l}$ & $\mathbf{2}$ \\
neutrophil & $>10000 / \mu \mathrm{l}$ & $\mathbf{1}$ \\
D-d & $>4 \mu \mathrm{g} / \mathrm{dl}$ & $\mathbf{1}$ \\
& $>8 \mu \mathrm{g} / \mathrm{dl}$ & 2 \\
& $>12 \mu \mathrm{g} / \mathrm{dl}$ & $\mathbf{3}$ \\
FXIII & $<75 \%$ & 1 \\
& $<45 \%$ & $\mathbf{2}$ \\
Na & $<136 \mathrm{mEq} / \mathrm{l}$ & $\mathbf{1}$ \\
Alb & $<3.8 \mathrm{~g} / \mathrm{dl}$ & $\mathbf{1}$ \\
& \multicolumn{2}{|c|}{ (Total 10 points) } \\
\hline
\end{tabular}

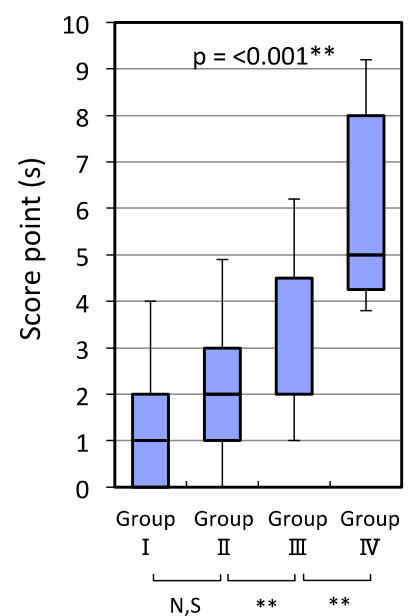

Figure 1 Parameters showed overall differences among Gl groups and construction of a scoring system. (A) WBC count, neutrophil count, serum albumin, potassium, plasma D-dimer and coagulation factor XIII levels in each Gl group are shown. These parameters showed overall significant differences by an analysis of variance (ANOVA) test. The probability values in the graph are for the ANOVA test, and those below the graph are for the Mann-Whitney $U$ test between consecutive groups. Bars in the graph represents the 10th, 25th, median, 75th, and 95th percentiles. (B) The scoring system consisting of the above parameters (left) and scores at the time of admission for each Gl group (right) are shown. The probability value in the graph is for the ANOVA test, and that below the graph are for the Mann-Whitney $U$ test between consecutive Gl groups. Bars in the graph represents the 10th, 25th, median, 75th, and 95th percentiles. 
Table 2 The average and standard deviation of each parameter and the scores for the Gl groups are shown

\begin{tabular}{|c|c|c|c|c|c|}
\hline mean $\pm S D$ & Group I & Group II & Group III & Group IV & $P$ value \\
\hline WBC count $(/ \mu l)$ & $8938 \pm 3057$ & $9575 \pm 3821$ & $10575 \pm 2993$ & $13808 \pm 4634$ & 0.004 \\
\hline neutrophil count $(/ \mu l)$ & $5068 \pm 2626$ & $6716 \pm 3661$ & $7324 \pm 3147$ & $10450 \pm 3879$ & 0.001 \\
\hline Albumin (g/dl) & $4.22 \pm 0.3$ & $4.19 \pm 0.5$ & $4.06 \pm 0.4$ & $3.64 \pm 0.5$ & 0.020 \\
\hline D-dimer ( $\mu \mathrm{g} / \mathrm{dl})$ & $3.72 \pm 3.6$ & $6.22 \pm 9.1$ & $8.75 \pm 9.0$ & $11.75 \pm 6.56$ & 0.012 \\
\hline Coagulation factor XIII (\%) & $87.34 \pm 26.5$ & $87.80 \pm 24.3$ & $72.12 \pm 25.2$ & $57.48 \pm 34.7$ & 0.001 \\
\hline Sodium (mEq)I & $138.86 \pm 2.1$ & $138.39 \pm 2.0$ & $137.36 \pm 2.1$ & $136.5 \pm 2.3$ & 0.044 \\
\hline Score Point(s) & $1.47 \pm 1.5$ & $2.17 \pm 1.8$ & $3.20 \pm 2.1$ & $5.8 \pm 2.4$ & $<0.001$ \\
\hline
\end{tabular}

for fecal hemoglobin), Group III: abdominal pain and positive for fecal hemoglobin, but not presenting with gross blood in the stools, and Group IV: abdominal pain with gross blood in the stools.

\section{Data analysis}

All analyses were carried out using the SPSS statistical software package, version 16.0 (SPSS, Chicago, IL, USA). Data are presented as the mean \pm standard deviation (SD) or median and range for continuous variables, or as the percentage of patients showing a given categorical variable. For all analyses, a 2-sided probability value below 0.05 was considered to indicate statistical significance. Spearman's rank correlation coefficient test was used to examine correlations among categorical parameters among the baseline characteristics and GI-involvement groups. A Kruskal-Wallis analysis of variance (ANOVA) was used to examine overall differences in laboratory data at the time of admission and other continuous variables among the GI-involvement groups. The data included WBC count, neutrophil count, hemoglobin, platelet count, serum concentration of albumin, aspartate aminotransferase (AST), alanine aminotransferase (ALT), lactate dehydrogenase $(\mathrm{LDH})$, and C-reactive protein $(\mathrm{CRP})$, prothrombin time (PT), activated partial thromboplastin time (APTT), plasma concentration of fibrinogen and D-dimer, coagulation factor XIII (fXIII) activity, serum concentration of antistreptolysin $\mathrm{O}$ antibody (ASO), IgA, complement C3, C4, total hemolytic complement (Ch50), sodium, and potassium. And age, days from symptom onset to admission, and days from abdominal pain onset to admission was also extracted. We then constructed a scoring model with which to identify severe GI involvement consisting of the parameters found to be statistically significant in the ANOVA analysis. A receiver operating characteristic (ROC) analysis was also carried out to examine the diagnostic utility of the constructed scoring model, and the Mann-Whitney $U$ test was used to compare scores between patients with and without arthralgia and nephritis. The correlation between duration of abdominal pain after admission and score at admission was also examined using Spearman's rank correlation coefficient.

\section{Results}

\section{Patient characteristics}

During the study period, 140 patients were analysed, with 27 later excluded (due to complications of intussusception

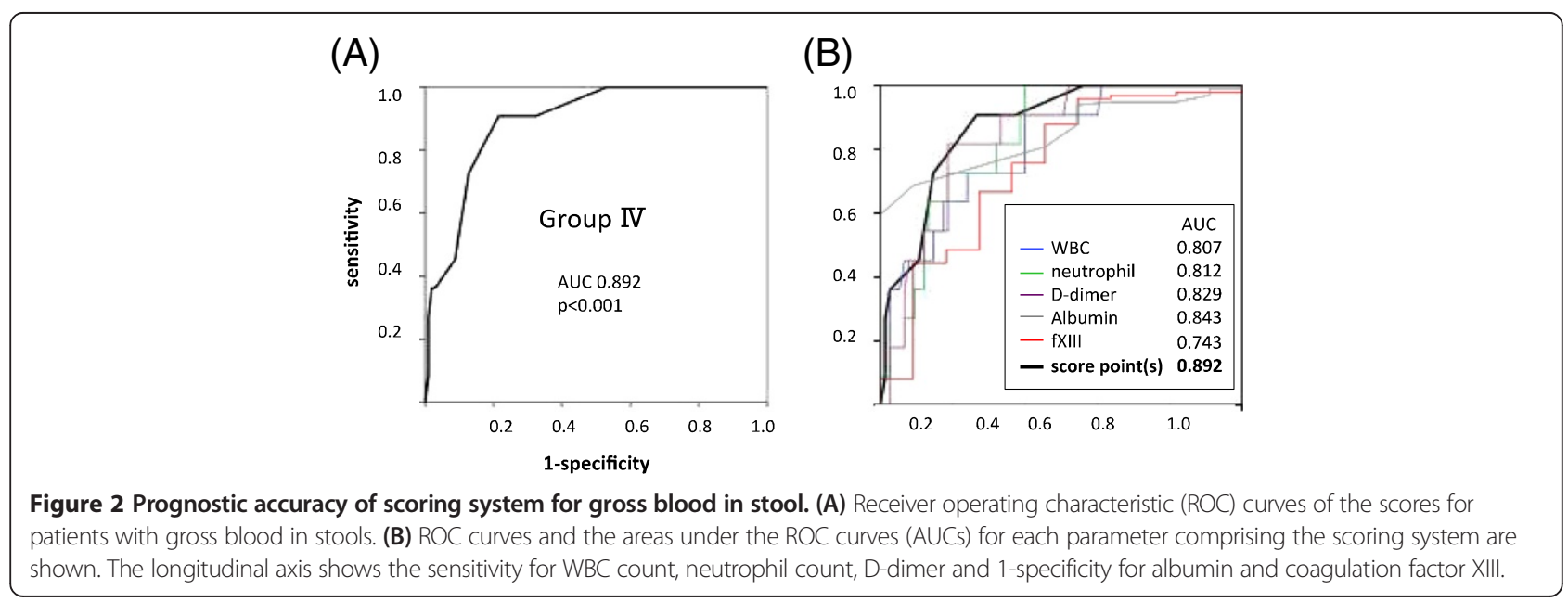




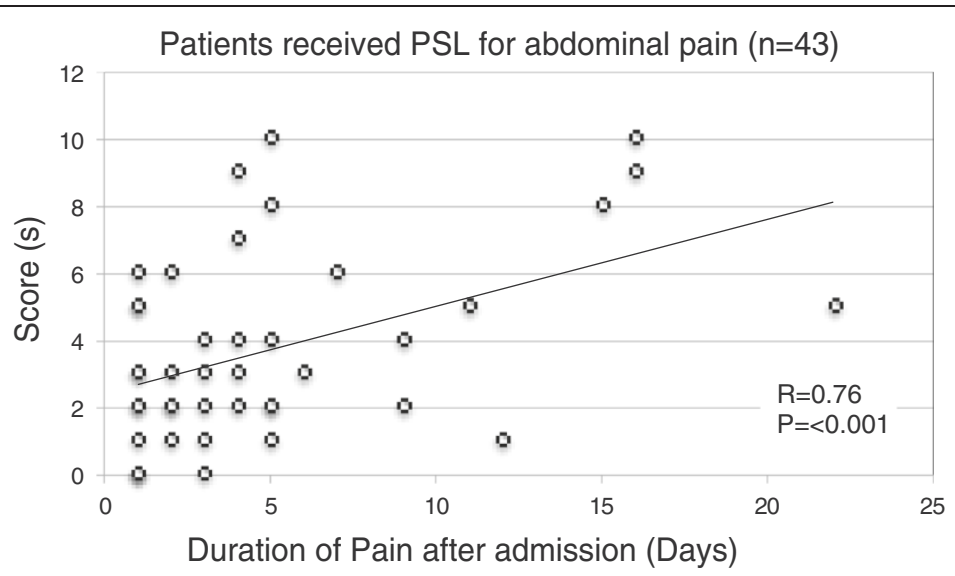

Figure 3 Association between initial score and subsequent duration of abdominal pain. Scatter plots of scores at the time of admission (longitudinal axis) and the subsequent duration of abdominal pain (horizontal axis) in 43 patients who received PSL therapy due to abdominal pain are shown.

in 3 , acute pancreatitis in 1 , testicular torsion in 1 , lack of data in 19, and presentation of abdominal pain later than two days after admission in 3 patients). Thus, 113 patients were included in the study. Table 1 shows the baseline characteristics of the patients by GI-involvement group. Although a significant correlation was observed between the development of HSPN and gender, with males more likely to develop HSPN, no significant differences were found among GI-involvement groups for any of the patient characteristics.

\section{Construction of a scoring system for the diagnosis of severe gastrointestinal involvement}

An ANOVA test showed the presence of significant overall differences in WBC count, neutrophil count, serum

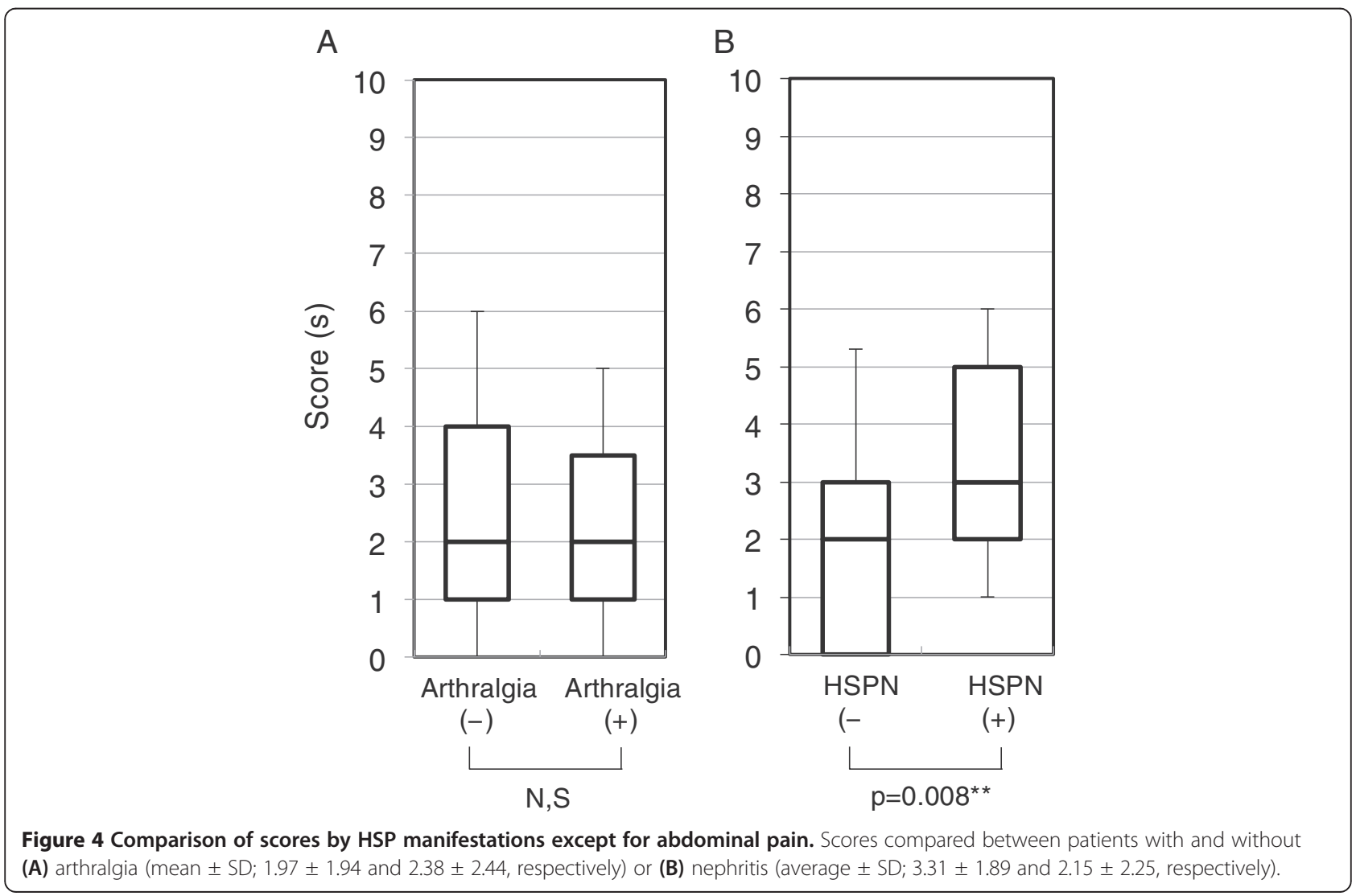


concentration of albumin, potassium, plasma level of D-dimer, and fXIII activity among the GI groups (Figure 1A). Construction of a scoring model consisting of these parameters was then carried out to increase prognostic utility (Figure $1 \mathrm{~B}$ ). The mean \pm SD of each parameter and score(s) are shown in Table 2. Subsequently, ROC curves for Group IV were also evaluated (Figure 2). The area under the ROC curve (AUC) of score(s) for Group IV patients demonstrated good prognostic accuracy (AUC $=0.892$ ). The construction of a scoring model improved diagnostic accuracy in comparison to that of each sole parameter in terms of AUC. To identify Group IV patients, the cut-off value was set at 4 points, a value which showed $90.0 \%$ sensitivity and $78.4 \%$ specificity.

\section{Association between initial score and subsequent duration of abdominal pain}

In the study population, 56 patients (49\%) presented with abdominal pain, placing them into Group II IV, and 43 of them (76\%) received PSL at $1.5 \sim 2 \mathrm{mg} / \mathrm{kg} /$ day. The scores at the time of admission for the 43 patients who received PSL for abdominal pain were significantly correlated with the subsequent duration of abdominal pain after admission (Figure 3).

\section{Association between initial score and subsequent development of HSPN}

A comparison of scores in association with manifestations of HSP excluding abdominal pain was also undertaken (Figure 4). There was no difference in scores between patients with or without arthralgia. A higher score was observed in patients with subsequent presentation of HSPN. However, the AUC for HSPN score showed only poor prognostic accuracy (AUC $=0.67$, the ROC curve is not shown).

\section{Discussion}

We focused on the prediction of significant GI involvement in the early phase of HSP, and found that our scoring system, consisting of generally available laboratory parameters, may be of value. A score of 4 points or higher, at the time of admission showed a good predictive value for gross blood in stools, and was associated with a longer duration of abdominal pain under PSL treatment. Based on its considerable predictive value for severe GI involvement, our scoring system has the potential to determine whether PSL intervention or supportive therapy, such as temporal fasting, should be undertaken. Further, as the monitoring of scores by repeat evaluation in several patients showed sequential changes parallel with GI symptoms (data not shown), it may also be useful for clinical follow up.
The precise mechanism underlying the changes in the parameters comprising the scoring system has not yet been clarified. Kawasaki disease, another systemic vasculitis common in children, often presents as hyponatremia and hypoalbuminemia, especially in severe cases (Fukunishi et al. 2000; Kobayashi et al. 2006). Further, patients with ulcerative colitis present with elevated D-dimer levels (Alkim et al. 2011; Zezos et al. 2009) and decreased fXIII activity (Lorenz et al. 1991), reflecting the inflammation of and injury to the intestinal mucosa. In consideration of these facts, our results might be regarded as reflecting severe vasculitis and the subsequent destruction of the small vessels, resulting in massive injury to the intestinal mucosa.

The efficacy of glucocorticoid therapy against the early symptoms of HSP remains controversial to a point. Several randomized controlled trials have reported that short-term PSL therapy $(1.5 \sim 2 \mathrm{mg} / \mathrm{kg} /$ day $)$ reduces the intensity of abdominal pain and arthralgia in early HSP (Ronkainen et al. 2006; Chartapisak et al. 2009; Rosenblum \& Winter 1987). However, there have been several patient reports describing severe, persistent GI involvement despite appropriate PSL therapy (Saulsbury 1999; Taylor et al. 1971). As the proportion of patients with severe GI involvement is small, their refractoriness might be hidden behind the majority of benign cases. However, in terms of preventing HSPN, early identification and management of this small proportion of patients with severe GI involvement might be more important as, along with persistent purpura (Kaku et al. 1998; Sano et al. 2002; Rigante et al. 2005; Shin \& Lee 2006; de Almeida et al. 2007), older age (Kaku et al. 1998; Sano et al. 2002; de Almeida et al. 2007), and decreased fXIII activity (Kaku et al. 1998; Sano et al. 2002), it is known to be an independent risk factor for HSPN (Kaku et al. 1998; Sano et al. 2002; Ronkainen et al. 2006; Shin \& Lee 2006). As current PSL therapy in early HSP does not prevent subsequent HSPN (Ronkainen et al. 2006; Chartapisak et al. 2009; Rosenblum \& Winter 1987; Huber et al. 2004), we propose the future evaluation of novel therapeutic strategies according to disease activity, and our scoring system might make an important contribution to this evaluation.

Of course, our study has several limitations. Our definition of GI group classifications was dependent on subjective symptoms and fecal examination; therefore, the classification criteria might require some improvement in terms of accuracy. Further, the proportion of patients with gross blood in stools was small. Last, our study was based on only a single cohort analysis. The survey of another cohort of HSP of HSP patients using our scoring system should help prove its validity. In addition, further large-scale or prospective studies are needed to overcome these limitations. 


\section{Conclusion}

The current study is the first report to the development of a scoring system for the identification of patients at risk for severe GI involvement on the basis of laboratory tests in the early phase of HSP.

\section{Competing interests}

The authors declare that they have no competing interests.

\section{Authors' contributions}

All of authors carried out the data collection. Data analysis and drafted the manuscript; TN and $\mathrm{OH}$. All authors discussed the results, read and approved the final manuscript.

\section{Acknowledgements}

We thank all investigators and their staff at the following institutions for their contributions to this study; Department of Pediatrics, Asahikawa Kosei Hospital; Department of Pediatrics, Nayoro City Hospital; Department of Pediatrics, Fukagawa City Hospital; Department of Pediatrics, Wakkanai City Hospital; Department of Pediatrics, Extended Mombetsu City Hospital; Department of Pediatrics, Engaru Kosei Hospital; and the Department of Pediatrics, Abashiri Kosei Hospital.

\section{Disclosure}

There are no prior publications or submissions with any overlapping information, including studies and patients.

\section{Author details}

'Department of Pediatrics, Asahikawa Medical University, Asahikawa, Hokkaido, Japan. ${ }^{2}$ Department of Pediatrics, Asahikawa Kosei Hospital, Asahikawa, Hokkaido, Japan. ${ }^{3}$ Department of Pediatrics, Nayoro City Hospital, Nayoro, Hokkaido, Japan. ${ }^{4}$ Department of Pediatrics, Fukagawa City Hospital, Fukagawa, Hokkaido, Japan. ${ }^{5}$ Department of Pediatrics, Wakkanai City Hospital, Wakkanai, Hokkaidō, Japan. ${ }^{6}$ Department of Pediatrics, Extended Mombetsu City Hospital, Mombetsu, Hokkaido, Japan. ${ }^{7}$ Department of Pediatrics, Abashiri Kosei Hospital, Abashiri, Hokkaido, Japan. ${ }^{8}$ Department of Pediatrics, Engaru Kosei Hospital, Hokkaido, Japan.

Received: 5 December 2013 Accepted: 21 March 2014 Published: 2 April 2014

\section{References}

Alkim H, Ayaz S, Alkim C, Ulker A, Sahin B (2011) Continuous active state of coagulation system in patients with nonthrombotic inflammatory bowel disease. Clin Appl Thromb Hemost 17(6):600-604

Bogdanovic R (2009) Henoch-Schonlein purpura nephritis in children: risk factors, prevention and treatment. Acta Paediatr 98(12):1882-1889

Calvino MC, Llorca J, Garcia-Porrua C, Fernandez-Iglesias JL, Rodriguez-Ledo P, Gonzalez-Gay MA (2001) Henoch-Schonlein purpura in children from northwestern Spain: a 20-year epidemiologic and clinical study. Medicine (Baltimore) 80(5):279-290

Chartapisak W, Opastiraku S, Willis NS, Craig JC, Hodson EM (2009) Prevention and treatment of renal disease in Henoch-Schonlein purpura: a systematic review. Arch Dis Child 94(2):132-137

Choong CK, Beasley SW (1998) Intra-abdominal manifestations of HenochSchonlein purpura. J Paediatr Child Health 34(5):405-409

de Almeida JL, Campos LM, Paim LB, Leone C, Koch VH, Silva CA (2007) Renal involvement in Henoch-Schonlein purpura: a multivariate analysis of initial prognostic factors. J Pediatr (Rio J) 83(3):259-266

Fukunishi M, Kikkawa M, Hamana K, Onodera T, Matsuzaki K, Matsumoto Y, Hara J (2000) Prediction of non-responsiveness to intravenous high-dose gamma-globulin therapy in patients with Kawasaki disease at onset. J Pediatr 137(2):172-176

Huber AM, King J, McLaine P, Klassen T, Pothos M (2004) A randomized, placebo-controlled trial of prednisone in early Henoch Schonlein Purpura [ISRCTN85109383]. BMC Med 2:7

Kaku Y, Nohara K, Honda S (1998) Renal involvement in Henoch-Schonlein purpura: a multivariate analysis of prognostic factors. Kidney Int 53(6):1755-1759

Kamitsuji H, Tani K, Yasui M, Taniguchi A, Taira K, Tsukada S, lida Y, Kanki H, Fukui $H$ (1987) Activity of blood coagulation factor XIII as a prognostic indicator in patients with Henoch-Schonlein purpura. Efficacy of factor XIII substitution. Eur J Pediatr 146(5):519-523

Kobayashi T, Inoue Y, Takeuchi K, Okada Y, Tamura K, Tomomasa T, Morikawa A (2006) Prediction of intravenous immunoglobulin unresponsiveness in patients with Kawasaki disease. Circulation 113(22):2606-2612

Lorenz R, Heinmuller M, Classen M, Tornieporth N, Gain T (1991) Substitution of factor XIII: a therapeutic approach to ulcerative colitis. Haemostasis 21(1):5-9

Narchi H (2005) Risk of long term renal impairment and duration of follow up recommended for Henoch-Schonlein purpura with normal or minimal urinary findings: a systematic review. Arch Dis Child 90(9):916-920

Rigante D, Candelli M, Federico G, Bartolozzi F, Porri MG, Stabile A (2005) Predictive factors of renal involvement or relapsing disease in children with Henoch-Schonlein purpura. Rheumatol Int 25(1):45-48

Ronkainen J, Koskimies O, Ala-Houhala M, Antikainen M, Merenmies J, Rajantie J, Ormala T, Turtinen J, Nuutinen M (2006) Early prednisone therapy in Henoch-Schonlein purpura: a randomized, double-blind, placebo-controlled trial. J Pediatr 149(2):241-247

Rosenblum ND, Winter HS (1987) Steroid effects on the course of abdominal pain in children with Henoch-Schonlein purpura. Pediatrics 79(6):1018-1021

Sano H, Izumida M, Shimizu H, Ogawa Y (2002) Risk factors of renal involvement and significant proteinuria in Henoch-Schonlein purpura. Eur J Pediatr 161 (4):196-201

Saulsbury FT (1993) Corticosteroid therapy does not prevent nephritis in Henoch-Schonlein purpura. Pediatr Nephrol 7(1):69-71

Saulsbury FT (1999) Henoch-Schonlein purpura in children. Report of 100 patients and review of the literature. Medicine (Baltimore) 78(6):395-409

Saulsbury FT (2001) Henoch-Schonlein purpura. Curr Opin Rheumatol 13(1):35-40

Saulsbury FT (2007) Clinical update: Henoch-Schonlein purpura. Lancet 369(9566):976-978

Shin Jl, Lee JS (2006) Prognostic serological markers of Henoch-Schonlein nephritis. QJM 99(11):801

Taylor WF, Amin LA, Mruthyunjaya GT (1971) Hypoalbuminemia in HenochSchonlein purpura following streptococcal infection. Description of a case in a child. Clin Pediatr (Phila) 10(12):737-739

Trapani S, Micheli A, Grisolia F, Resti M, Chiappini E, Falcini F, De Martino M (2005) Henoch Schonlein purpura in childhood: epidemiological and clinical analysis of 150 cases over a 5-year period and review of literature. Semin Arthritis Rheum 35(3):143-153

Yilmaz D, Kavakli K, Ozkayin N (2005) The elevated markers of hypercoagulability in children with Henoch-Schonlein purpura. Pediatr Hematol Oncol 22(1):41-48

Zezos P, Papaioannou G, Nikolaidis N, Patsiaoura K, Vassiliadis T, Mpoumponaris A, Giouleme O, Evgenidis N (2009) Elevated markers of thrombin generation and fibrinolysis in patients with active and quiescent ulcerative colitis. Med Sci Monit 15(11):CR563-CR572

Zhang Y, Huang X (2008) Gastrointestinal involvement in Henoch-Schonlein purpura. Scand J Gastroenterol 43(9):1038-1043

doi:10.1186/2193-1801-3-17

Cite this article as: Nagamori et al: Construction of a scoring system for predicting the risk of severe gastrointestinal involvement in Henoch-Schönlein Purpura. SpringerPlus 2014 3:171.

\section{Submit your manuscript to a SpringerOpen ${ }^{\odot}$ journal and benefit from:}

- Convenient online submission

Rigorous peer review

- Immediate publication on acceptance

- Open access: articles freely available online

- High visibility within the field

- Retaining the copyright to your article

Submit your next manuscript at $>$ springeropen.com 